\title{
Radiologically Documented Enlargement of Retro-Cerebellar Arachnoid Cyst Causing Obstructive Hydrocephalus in Late Adulthood. A Case Report
}

\author{
Florence Constance Maria Reith, MD, PhD ${ }^{1}$, Markus Schmidt, MBBS(Hons), FANZCA ${ }^{1,2}$ and Gabriel Yin \\ Foo Lee, MBBS(Hons), MS, FRACS ${ }^{1,2,3^{*}}$
}

${ }^{1}$ Sir Charles Gairdner Hospital, Perth, Australia

${ }^{2}$ Saint John of God Hospital, Subiaco, Australia

${ }^{3}$ School of Surgery, University of Western Australia, Australia

*Corresponding author: Gabriel Yin Foo Lee, Hospital Ave, Sir Charles Gairdner Hospital; Saint John of God Hospital, Subiaco; School of Surgery, University of Western Australia, WA 6009, Australia

\begin{abstract}
Background: Intracranial arachnoid cysts $(A C)$ rarely enlarge and become symptomatic, especially in adults and when located retrocerebellar. This report describes an adult in which an expanding retrocerebellar arachnoid cyst prompted surgical resection and it provides an overview of the literature and recommendations for optimal management.

Case presentation: A 65-year-old women was diagnosed with an incidental retrocerebellar $A C$. She presented 10 years later with headaches, aural fullness and poor balance. Consecutive MRI showed significant enlargement of the AC, associated with tri-ventricular hydrocephalus. She underwent cervico-cranial decompression, cyst fenestration and shunting. Her symptoms improved significantly. Post-operative MRI showed reduction in AC size and resolution of hydrocephalus.
\end{abstract}

Conclusions: In adults, only a minority of ACs will enlarge and become symptomatic prompting surgical intervention. An optimal approach to management should focus on counseling around alarming symptoms rather than continual follow-up.

\section{Keywords}

Arachnoid cyst, Adults, Enlargement, Surgical treatment, Case report

\section{Background}

Intracranial arachnoid cysts are benign conditions. Arachnoid cysts are believed to exemplify a congenital abnormality which arises from splitting or duplication of the arachnoid layer of the meninges during embryonic development $[1,2]$. However, secondary intracranial arachnoid cysts have been reported to develop de novo $[3,4]$ or can be related to head trauma, [5] infection, [6] and intraventricular haemorrhage of prematurity [7].

Commonly, arachnoid cysts are incidental findings and asymptomatic. A retrospective case review found that over $87 \%$ of patients with arachnoid cysts were asymptomatic and that symptomatic patients tend to be younger and more likely have associated hydrocephalus [8]. The natural history of arachnoid cysts is, however, not well understood. In paediatric patients, it was found that arachnoid cysts can enlarge, decrease in size or spontaneously resolve over time. Also, the overall behaviour was more likely to be benign with clinical or radiological stability over time in children who were older at the time of initial presentation [9]. In line with this, in the adult population, documented growth of an intracranial arachnoid cyst is a rare entity. This case report describes a case of a posterior fossa cerebellar arachnoid cyst that became symptomatic with evidence of

Citation: Reith FCM, Schmidt M, Lee GYF (2021) Radiologically Documented Enlargement of Retro-Cerebellar Arachnoid Cyst Causing Obstructive Hydrocephalus in Late Adulthood. A Case Report. Neurosurg Cases Rev 4:066. doi.org/10.23937/2643-4474/1710066

Accepted: April 21, 2021; Published: April 23, 2021

Copyright: (C) 2021 Reith FCM, et al. This is an open-access article distributed under the terms of the Creative Commons Attribution License, which permits unrestricted use, distribution, and reproduction in any medium, provided the original author and source are credited. 


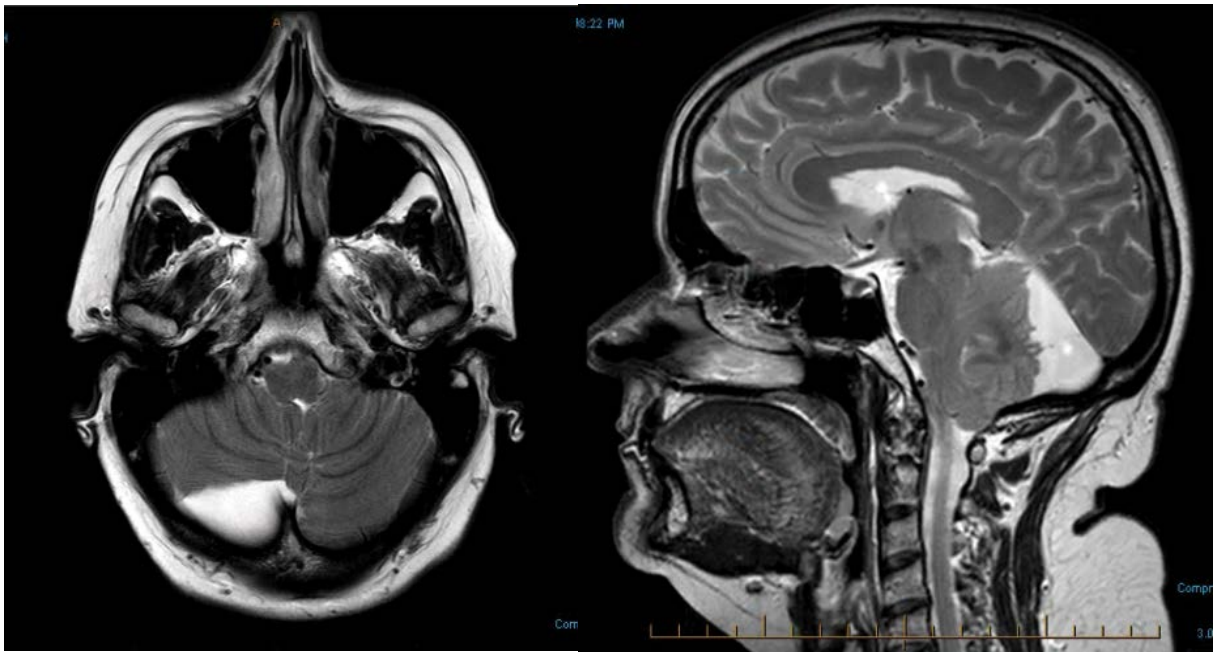

Figure 1: MRI showing initial diagnosis of retrocerebellar arachnoid cyst in 2008.

Figure 1a: T2 weighted axial MR image showing a right retrocerebellar arachnoid cyst, measuring $19 \mathrm{~mm}$ anteroposterior, $39 \mathrm{~mm}$ transversely and $40 \mathrm{~mm}$ craniocaudal at timing of initial diagnosis in 2008.

Figure 1b: T2 weighted sagittal MR image revealing cerebellar tonsillar ectopia of $4 \mathrm{~mm}$ associated with crowding of the neural structures at the foramen magnum, but no hydrocephalus.

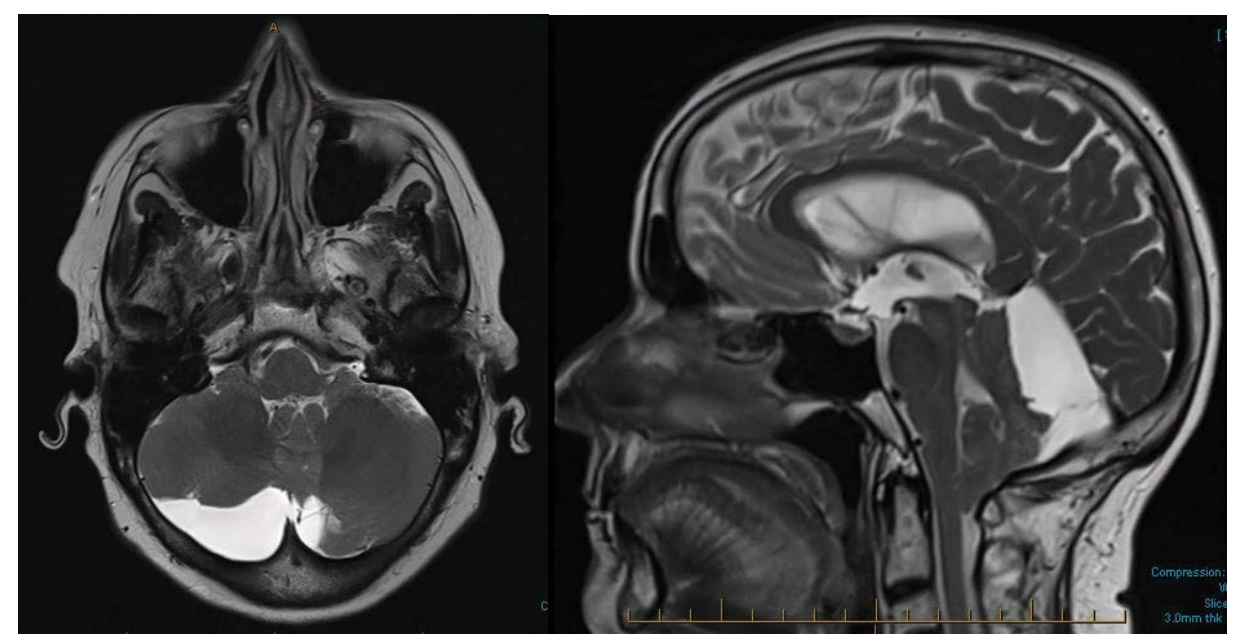

Figure 2: MRI findings showing significant increase in size of the retrocerebellar arachnoid cyst 12 years later.

Figure 2a: T2 weighted axial MR image reveals that the arachnoid cyst now measures $25 \mathrm{~mm}$ anteroposterior, $67 \mathrm{~mm}$ transversely, $56 \mathrm{~mm}$ craniocaudal, with significant mass effect on the posterior fossa structures.

Figure 2b: T2 weighted sagittal MR image showing a $11 \mathrm{~mm}$ descent of the right cerebellar tonsil through the foramen magnum. There is transtentorial herniation with some effacement of quadrigeminal cistern. There is supratentorial ventricular dilatation, and periventricular hyperintensity suggesting transependymal CSF seepage.

documented expansion of the cyst and associated development of hydrocephalus and Chiari malformation.

\section{Case Presentation}

The authors report a sixty-five year old woman of excellent general health who had presented with intermittent occipital headaches and dizziness initially about 12 years ago. Her MRI brain scan disclosed a posterior fossa arachnoid cyst and borderline Chiari malformation (Figure 1). She was assessed by a neurologist. The arachnoid cyst was thought to be an incidental finding and she was deemed to have cervicogenic headaches. The symptoms were managed conservatively and remained stable until 12 years later, when the patient experienced new symptoms of aural "fullness" and poor balance. While walking along a straight path, she was observed to intermittently veer off in an unexpected direction but always managing to correct herself without falling over. Neurological examination showed no focal deficits. There were no signs of cerebellar dysfunction or truncal ataxia. Repeat MRI imaging demonstrated significant enlargement of the retro-cerebellar arachnoid cyst and increased mass effect on the cerebellum (Figure 2). The compression of the aqueduct was associated with tri-ventricular hydrocephalus. The combination of clinical symptoms and radiological findings prompted to surgical management. 


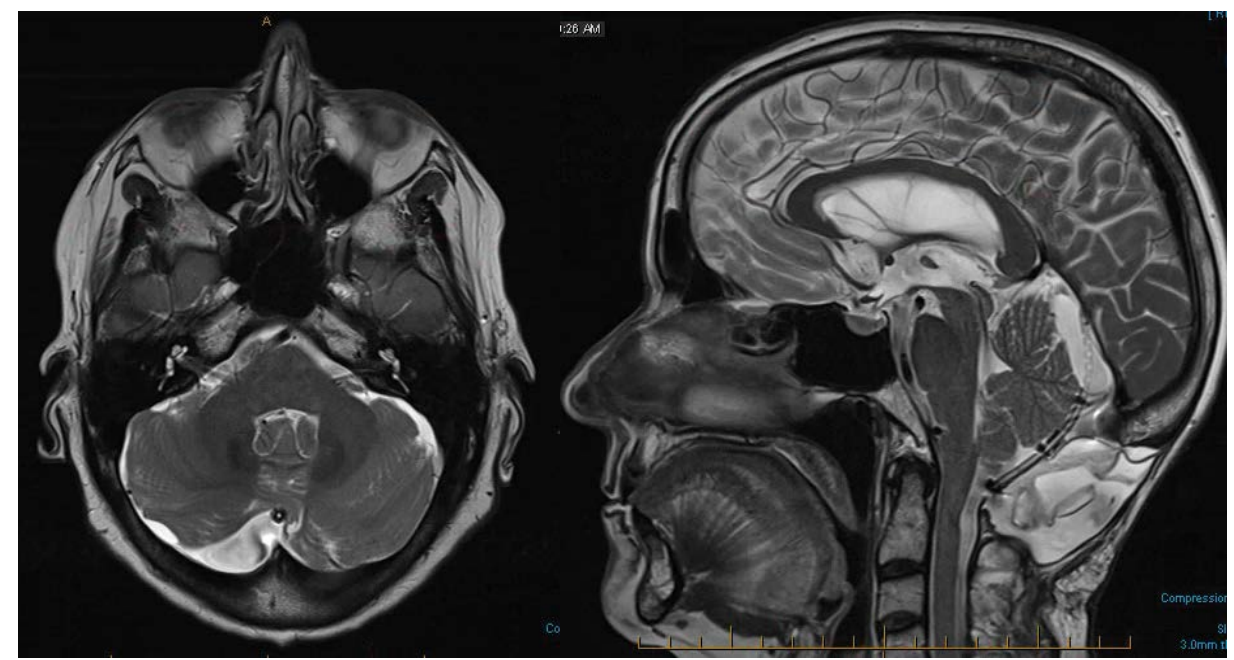

Figure 3: Postoperative MRI findings.

Figure 3a: T2 weighted axial MR image.

Figure 3b: T2 weighted sagittal MR image of the postoperative status. The mass effect of the retrocerebellar cyst has been ameliorated, without any tonsillar descent present. There is a reduction of the triventricular hydrocephalus. Cystosubarachnoid shunt present from residual arachnoid cyst to level of C2.

Thepatientunderwentcranio-cervicaldecompression surgery. The posterior rim of the foramen magnum and a two $\mathrm{cm}$ zone of occipital skull was resected with the posterior arch of $\mathrm{C} 1$. The dura was particularly thin and friable. A Y-shaped durotomy was performed to expose the arachnoid cyst and extended inferiorly beyond the cerebellar tonsils. The arachnoid cyst membrane was widely fenestrated. A cyst-spinal arachnoid shunt was also placed. The patient recovered uneventfully.

On review at four months post-operatively, the patient experienced significant improvement of her symptoms. Her balance was notably improved. Followup MR imaging demonstrated significant reduction in size of the arachnoid cyst with resolution of the hydrocephalus (Figure 3).

\section{Discussion and Conclusions}

Intracranial arachnoid cysts are common incidental conditions with a reported prevalence of $2.6 \%$ and $1.4 \%$ in children and adults, respectively $[9,10]$. A MRI study in 2,536 healthy young male applicants for military flying duties, showed a similar prevalence of $1.7 \%$ [11]. Documented enlargement of arachnoid cysts in adults is, however, rare.

Al-Holou, et al. studied the natural history of arachnoid cysts in 203 adults that had 213 arachnoid cysts. The majority of arachnoid cysts in this series remained stable in size $(94.4 \%)$, whereas 5 cysts $(2.3 \%)$ enlarged during the follow up period (minimum 6 months) with a mean growth of $0.4 \mathrm{~cm}$ in each dimension. These included 3 middle fossa cysts and 2 convexity cysts. Only one of these patients $(0.5 \%)$ with an enlarging convexity arachnoid cyst, developed new symptoms, including headache and seizure. After fenestration, the symptoms improved and the cyst size reduced. None of the predictive factors (including age, sex, initial cyst size or location) were found to be significantly related to cyst expansion on logistic regression analysis [10].

Another retrospective research study reviewed 369 adults with arachnoid cysts of which $92.4 \%$ were asymptomatic. 25 of the 28 symptomatic patients underwent surgical intervention on initial presentation, which represents $6.8 \%$ of all adult arachnoid cysts. None of the patients in this series showed enlargement of the arachnoid cyst on follow up imaging. Most of the cysts remained unchanged (95\%), whereas the rest reduced or resolved [8]. A different picture is reflected in the pediatric population. Al-Holou, et al. described that $10 \%$ of 309 children showed extension of their arachnoid cyst [9]. The rate of symptomatic increase in cyst size in pediatric population is documented to vary between $0.7 \%$ to $2.7 \%[9,12]$. These findings suggest that cyst expansion is related to younger age and that clinical deterioration from asymptomatic cysts is very unusual in adults. It was speculated that this finding is related to the fact that ageing might change the CSF dynamics or the arachnoid cyst architecture which stabilizes cyst size [8]. This finding, however, also calls into question the need for routine follow up using sequential MRI in asymptomatic adults.

Nevertheless, the specific location of the intracranial arachnoid cyst should be taken into account when considering frequency and modality of follow up. Whereas the majority of all intracranial arachnoid cysts are located in the middle fossa in the adult population, the symptomatic intracranial cysts are often located suprasellar, $[8,10]$ intraventricular, quadrigeminal cistern, [8] CPA and ambient cistern [10]. Whether these arachnoid cysts which are located within or in close proximity to major CSF cisterns enlarge because of different 
pathophysiological mechanisms remains speculative. Regardless, arachnoid cysts in these locations are more likely to cause obstructive hydrocephalus by obstructing normal CSF flow pathways, leading to symptoms related to hydrocephalus and increased intracranial pressure. Rarely, sudden changes in the cyst architecture causes symptoms due to acute cyst expansion, sudden hemorrhage into the cyst [13] and rupture of the cyst into the subdural space causing subdural hygromas or subdural hemorrhages [14].

Hall, et al. found that the most common presenting symptoms in the adult symptomatic group included headache, cranial nerve dysfunction (mostly visual symptoms, hearing loss, facial nerve palsy) and nausea/vomiting [8]. In the study of Al-Holou, et al. 5.3\% of adult patients with intracranial arachnoid cysts were symptomatic, and $3.6 \%$ of their population underwent surgery. They mainly presented with headache, gait imbalance, hydrocephalus, seizures, vertigo, visual disturbances, nausea/vomiting, hearing loss, speech abnormalities and cervical myelopathy with syrinx. Moreover, symptomatic cyst were related to younger mean age (42 years versus 49.5 years) and larger cyst size [10].

The pathophysiological basis for arachnoid cyst enlargement remains enigmatic. Several theories have been proposed, including an osmotic gradient, [15] fluid secretion by cells lining the cyst wall, $[16,17]$ and an one-way valve mechanism pushing fluid into the cyst with pulsations of cerebrospinal fluid [18]. A prospective study focusing on the cyst-wall morphology of arachnoid cysts demonstrated variability of epithelial linings and extracellular components suggesting different barrier properties and fluid turnover characteristics, making arachnoid cysts a heterogeneous group of pathological conditions [19]. The varying morphology and potential different underlying etiological mechanisms could be relevant in identifying arachnoid cysts that will enlarge and become symptomatic over time.

This case report, though it was stated that arachnoid cysts both in the middle fossa and retrocerebellar locations are less likely to be symptomatic, [10] illustrates how an arachnoid cyst in the retrocerebellar location became symptomatic over time with enlargement of the cyst size causing obstructive hydrocephalus and Chiari malformation. And although symptomatic enlargement of intracranial arachnoid cyst in adulthood is a known entity, it is only rarely documented [10]. Further research, focusing on the natural history, morphology and characteristics of arachnoid cysts, might help to identify adults at risk for cyst expansion and can lead to more focused follow up and treatment in this specific patient group.

Although the literature suggests that the risk of arachnoid cyst enlargement in adults is definite, it is very small. Perpetual follow up in this patient group therefore would lead to potential unnecessary long term follow up with regular imaging studies performed with a questionable cost-effectiveness. An optimal approach to clinically manage these patients should perhaps focus around accurate counselling of the patient, thereby explaining the risk and associated symptoms of cyst enlargement. Accordingly, the patient can present appropriately for clinical and imaging re-assessment when either symptoms worsen, or new symptoms develop.

\section{Declarations}

\section{Ethical approval and consent to participate}

All procedures performed were in accordance with the ethical standards of the institutional and/or national research committee and with the 1964 Helsinki declaration and its later amendments or comparable ethical standards. The Sir Charles Gairdner and Osborne Park Health Care Group Human Research Ethics Committee (HREC) approved publication of this case report on $8^{\text {th }}$ of December 2020. A copy of the approval form is available for review by the Editor of this journal.

Informed consent was obtained from the participant included in the study.

\section{Consent for publication}

Written informed consent for publication of their clinical details and/or clinical images was obtained from the patient. A copy of the consent form is available for review by the Editor of this journal.

\section{Availability of data and materials}

Data sharing not applicable to this article as no datasets were generated or analysed during the current study.

\section{Competing interests}

The authors declare that they have no conflict of interest.

\section{Funding}

No funding was received for this research.

\section{Authors' contributions}

GYFL and MS were involved in management and treatment of the patient and provided input and writing for the case report. FR performed a literature research on the topic and was a major contributor in writing the manuscript. All authors read and approved the final manuscript.

\section{Acknowledgements}

Not applicable

\section{References}

1. Starkman SP, Brown TC, Linell EA (1958) Cerebral arachnoid cysts. J Neuropathol Exp Neurol 17: 484-500. 
2. Rengachary SS, Watanabe I, Brackett CE (1978) Pathogenesis of intracranial arachnoid cysts. Surg Neurol februari 9: 139-144.

3. Brewington D, Petrov D, Whitmore R, Liu G, Wolf R, et al (2017) De novo intraneural arachnoid cyst presenting with complete third nerve palsy: Case report and literature review. World Neurosurg 98.

4. Invergo D, Tomita T (2012) De novo suprasellar arachnoid cyst: Case report and review of the literature. Pediatr Neurosurg 48: 199-203.

5. Ibrahim GM, Alotaibi NM, Lipsman N, Nassiri F, da Costa L (2017) De novo formation of a symptomatic arachnoid cyst in an adult. Neurology 88: 331-332.

6. Clifton W, Rahmathulla G, Tavanaiepour K, Alcindor D, Jakubek G, et al. (2018) Surgically treated de novo cervicomedullary arachnoid cyst in symptomatic adult patient. World Neurosurg 116: 329-332.

7. Palin M, Anderson I, O'Reilly G, Goodden JR (2015) A suprasellar arachnoid cyst resulting from an intraventricular haemorrhage and showing complete resolution following endoscopic fenestration. BMJ Case Rep.

8. Hall S, Smedley A, Sparrow O, Mathad N, Waters R, et al. (2019) Natural history of intracranial arachnoid cysts. World Neurosurg 126: e1315-e1320.

9. Al-Holou WN, Yew AY, Boomsaad ZE, Garton HJL, Muraszko KM, et al. (2010) Prevalence and natural history of arachnoid cysts in children. J Neurosurg Pediatr 5: 578585.

10. Al-Holou WN, Terman S, Kilburg C, Garton HJL, Muraszko $\mathrm{KM}$, et al. (2013) Prevalence and natural history of arachnoid cysts in adults. J Neurosurg 118: 222-231.

11. Weber F, Knopf H (2006) Incidental findings in magnetic resonance imaging of the brains of healthy young men. $J$ Neurol Sci 240: 81-84.
12. Huang J-H, Mei W-Z, Chen Y, Chen J-W, Lin Z-X (2015) Analysis on clinical characteristics of intracranial Arachnoid Cysts in 488 pediatric cases. Int J Clin Exp Med 8: 1834318350.

13. Adin ME, Yıldız MS, Deniz MA, Behzadi AH, Mata-Mbemba D (2018) Arachnoid cysts with spontaneous intracystic hemorrhage and associated subdural hematoma: Report of management and follow-up of 2 cases. Radiol Case Rep 13: 516-521.

14. Mori K, Yamamoto T, Horinaka N, Maeda M (2002) Arachnoid cyst is a risk factor for chronic subdural hematoma in juveniles: Twelve cases of chronic subdural hematoma associated with arachnoid cyst. J Neurotrauma 19: 10171027.

15. Sandberg DI, McComb JG, Krieger MD (2005) Chemical analysis of fluid obtained from intracranial arachnoid cysts in pediatric patients. J Neurosurg 103: 427-432.

16. Go KG, Houthoff HJ, Blaauw EH, Havinga $P$, Hartsuiker J (1984) Arachnoid cysts of the sylvian fissure. Evidence of fluid secretion. J Neurosurg 60: 803-813.

17. Helland CA, Aarhus M, Knappskog P, Olsson LK, Lund-Johansen M, et al. (2010) Increased NKCC1 expression in arachnoid cysts supports secretory basis for cyst formation. Exp Neurol 224: 424-428.

18. Halani SH, Safain MG, Heilman CB (2013) Arachnoid cyst slit valves: The mechanism for arachnoid cyst enlargement. J Neurosurg Pediatr 12: 62-66.

19. Rabiei K, Tisell M, Wikkelso C, Johansson BR (2014) Diverse arachnoid cyst morphology indicates different pathophysiological origins. Fluids Barriers CNS 11: 5. 